\title{
Acute augmentation for interstitial insufficiency of the posterior cruciate ligament. A two to five year clinical and radiographic study
}

\author{
Terence Wai-kit Chan ${ }^{1}$ \\ Chi-Chung Kong ${ }^{1}$ \\ Angelo Del Buono ${ }^{2}$ \\ Nicola Maffulli ${ }^{3,4}$
}

Investigation performed at North District Hospital, Hong Kong

\footnotetext{
1 Asia Medical Specialists, Hong Kong

2 Department of Orthopaedic and Trauma Surgery, Hospital Vaio, Fidenza, Italy

3 Department of Musculoskeletal Disorders, University of Salerno School of Medicine, Salerno, Italy

4 Centre for Sports and Exercise Medicine, Barts and The London School of Medicine and Dentistry, Mile End Hospital, London, UK
}

Corresponding author:

Nicola Maffulli

Centre for Sports and Exercise Medicine, Barts and The London School of Medicine and Dentistry, Mile End Hospital

275 Bancroft Road, London E1 4DG, UK

E-mail: n.maffulli@qmul.ac.uk

\section{Summary}

Background: there is need to ascertain clinical and imaging outcomes after posterior cruciate ligament (PCL) augmentation.

Methods: we performed a retrospective analysis of clinical, imaging and functional data on 21 physically active males who underwent arthroscopic trans-tibial augmentation of the PCL for symptomatic grade III PCL insufficiency. The average follow-up time was 50 months (24-60 months). The Lysholm knee score was administered to all the patients, ligament laxity was evaluated with the posterior drawer test, the KT-1000 arthrometer, and the anteromedial tibial step-off. Standing antero-posterior, lateral and Merchant's view radiographs were taken preoperatively and at annual follow-up.

Results: post-operatively, ligament laxity and Lysholm knee scores were significantly improved than at baseline. Sixteen patients $(73 \%)$ returned to pre-injury sport activity level, 3 patients (14\%) returned to a lower level, 2 had to stop. We found radiographic degenerative changes in 5 of 22 affected knees $(23 \%)$, with evidence of a statistically significant association between the occurrence of degenerative changes and the interval time from injury to surgery and duration of the follow up.

Conclusions: arthroscopic transtibial single bundle autograft hamstring augmentation significantly improves the function of the knee, with an overall satisfactory outcome of $\mathbf{8 2} \%$ at $\mathbf{2 - 5}$ years from surgery.

KEY WORDS: PCL, arthroscopic augmentation, clinical outcomes, imaging.

\section{Introduction}

Management of isolated grade III injuries of the posterior cruciate ligament (PCL) is controversial. Historically, conservative measures have provided good results in most patient ${ }^{1-3}$, however the increasing understanding of the natural history of PCL insufficiency has induced many surgeons to recommend reconstruction or augmentation to prevent decline of the knee function in the short term, and the development of osteoarthritis of the patello-femoral joint and the medial compartment in the long term ${ }^{4}$, secondary to the damage to cartilage and meniscal tears ${ }^{5-9}$.

Surgical reconstruction is usually recommended in symptomatic patients with grade III PCL tears who complain of pain and instability, and in those with multiple ligament injuries. In this decision making process, some concerns regard the optimal time for surgery. We hypothesized that a longer delay from injury to surgery negatively affects outcome.

\section{Materials and methods}

This is a retrospective analysis of clinical, imaging and functional data on 21 physically active males who underwent arthroscopic augmentation of the PCL for symptomatic grade III PCL insufficiency. The patients underwent surgery at an average age of 28 years (19 to 52), after an average period of 5.6 months from the traumatic episode (range 1 to 25 months). Inclusion criteria were grade III insufficiency of the PCL. Patients with systemic diseases, neuromuscular disorders, obesity, marked anatomic deformities, and previous surgery on the index knee were excluded from this study. We also excluded patients with PCL avul- 
Acute augmentation for interstitial insufficiency of the posterior cruciate ligament. A two to five year clinical and radiographic study

sion fractures and multiple ligament injuries. The left knee was affected in 12 patients, the right knee in 10. One patient sustained bilateral PCL injury, complicated by fracture of both femur.

Causes of injury were vehicle accidents in 14 patients and sports injuries in 7. Associated injuries were femoral fractures in 2, tibial fracture in 1, upper limb fractures in 3 , and meniscal tears in 3 patients. The average follow-up time was 50 months (36-72 months). All procedures described in the present investigation were approved by the local ethics committee.

\section{Surgical procedure}

All patients were operated in the supine position by a single fully trained orthopaedic surgeon (CCK), under general or epidural anesthesia using a standard setup. After exsanguination, a thigh tourniquet was inflated to $250 \mathrm{mmHg}$, and a $50 \mathrm{mmHg}$ arthroscopic pump was used for irrigation. Associated injuries were identified and treated before PCL reconstruction. The concomitant procedures included one medial partial menisectomy, 3 meniscal repairs, 1 lateral retinaculum release, and 4 debridements for osteoarthritic changes involving the medial and patellofemoral compartments.

Arthroscopic transtibial single bundle PCL augmentation was performed using a standard technique ${ }^{10}$ and quadrupled hamstrings autografts. Viewing with a $70^{\circ}$ arthroscope through the anteromedial portal and a working instrument in the posteromedial portal, the anatomical attachment of the PCL at both tibial and femoral side was identified and the PCL remnant debrided of synovium and scar tissue, taking care to preserve it. The entry point of the tibial tunnel was approximately 4-5 cm below the joint line, on the anteromedial aspect of the tibia; the exit point was on the tibial footprint, more proximally and laterally than the entry point. A $2.2 \mathrm{~mm}$ guide pin was inserted under fluoroscopic monitoring, to better assess its position, and a cannulated drill of the diameter of the graft was used to drill the tibial tunnel. The progression of the drill is monitored under fluoroscopy, and the neurovascular bundle protected by the surgeon's finger tip (Fig. 1). The anatomical femoral footprint of the $\mathrm{PCL}$ posterolateral bundle was identified, a $2.2 \mathrm{~mm}$ guide pin was inserted intra-articularly through the femoral PCL footprint, overdrilled with a cannulated Endobutton drill (Smith \& Nephew), and a femoral socket of the same diameter as the graft drilled to leave $10 \mathrm{~mm}$ of intact condyle as a bone bridge.

The average diameter of the graft was $9 \mathrm{~mm}$; the average length was $127 \mathrm{~mm}$, ranging from 115 to $138 \mathrm{~mm}$.

The graft was passed in a standard fashion from tibia to femur and proximal fixation performed with an endobutton (Smith \& Nephew) and a bioabsorbable interference screw (Bioscrew, Linvatec) of the same diameter as the tunnel.

Pretension of the graft was performed by 20 cycles of flexion and extension of the knee. A normal $10 \mathrm{~mm}$

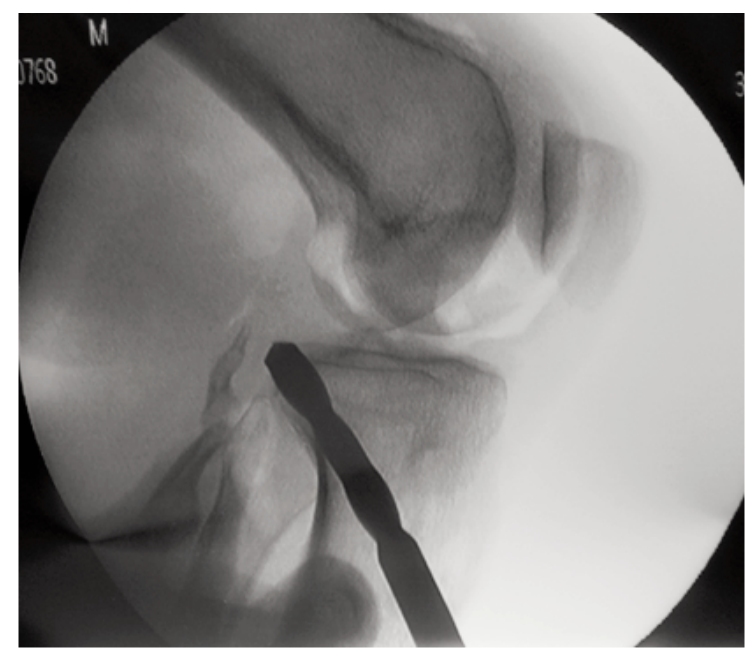

Figure 1. Protection of the posterior structures of the kneee by surgeon's finger tip through posteromedial safety incision as suggested by Fanelli.

anteromedial step-off of the tibia was restored by anterior draw at $90^{\circ}$ of knee flexion. A screw post and a bioabsorbable interference screw (Bioscrew or Extraloc, Linvatec) of diameter $1 \mathrm{~mm}$ larger than the tunnel were used for tibial graft fixation.

\section{Postoperative rehabilitation}

Patients undertook a standardised postoperative rehabilitation protocol under the supervision of physiotherapist: the knee was braced allowing $0-60^{\circ}$ for four weeks, and partial weight-bearing walking, range of motion exercises, and quadriceps strengthening exercises were commenced immediately. Hamstring exercises were allowed after 6 weeks.

\section{Outcome evaluation}

Patients were assessed thereafter at three month intervals for one year, and yearly thereafter.

To assess the functional status, the Lysholm knee score was administered to all the patients. Ligament laxity was evaluated clinically with the posterior drawer test at $90^{\circ}$ of flexion, and with the KT-1000 arthrometer. Anteromedial tibial step-off was also assessed. Standing antero-posterior, lateral and Merchant's view radiographs were taken preoperatively and at annual follow-up. Radiographic findings were categorised according to the Ahlbäck's classification $^{11}$. Pre- and postoperative data were compared using the Wilcoxon Signed Rank test. The correlation of clinical outcome and secondary osteoarthritis of the affected knees with the duration of injury, grading of residual post-operative PCL laxity and the length of follow-up time were evaluated using the Mann-Whitney test, with statistical significance set at $P<0.05$. 


\section{Results}

There were no intra-operative complications. Haematoma at the graft donor site occurred in one patient; one patient underwent manipulation under anesthesia and arthroscopic lysis for postoperative arthrofibrosis, and a full range of motion was regained.

\section{Functional assessment}

The symptoms reported by the patients are summarised in Table 1. Preoperatively, pain and instability were present in $59.1 \%(13 / 22)$ of the knees, only pain in $18.2 \%(4 / 22)$ and only instability in $22.7 \%$ (5/22). Postoperatively, pain and instability persisted in $4.5 \%(1 / 22)$ knees, $1(4.5 \%)$ patient complained of only pain, $2(9.1 \%)$ patients of only instability. The functional improvement was statistically significant $(P<0.001)$. From the analysis of the relationship of symptoms, Lysholm score and time from injury to surgery (Tab. 2), patients were less symptomatic and more satisfied when operated on within 6 months from the injury $(P<0.05)$. The average Lysholm scores improved from $49 \pm 17$ pre-operatively to $86 \pm 7$ postoperatively $(P<0.001)$. Six knees $(27.3 \%)$ were scored as excellent ( $\geq 95), 12(54.6 \%)$ as good (8594), $3(13.6 \%)$ as fair (65-84), and $1(4.5 \%)$ as poor $(\leq 65)$. Sixteen patients $(72.7 \%)$ returned to pre-injury sport activity level, 3 patients $(13.6 \%)$ returned to a lower level, 2 had to stop. Overall, 14 (63.6\%) patients were satisfied with the functional outcomes.

\section{Ligament laxity}

Pre-operatively, posterior laxity greater than $10 \mathrm{~mm}$ was diagnosed in all knees, but none of them presented significant postero-lateral laxity (Tab. 3). Postoperatively, we found no laxity in $27.3 \%(6 / 22)$ of the knees, grade I laxity with a firm end point $(0-5 \mathrm{~mm})$ in $54.5 \%(12 / 22)$, and grade II (5-10 mm) laxity with firm end point on posterior drawer test in $18.2 \%(4 / 22)$. Comparing pre-operative and post-operative status, we found a statistically significant improvement in ligament laxity $(P<0.001)$.

\section{Radiographic assessment}

We found radiographic degenerative changes in 5 of 22 affected knees (22.7\%): Ahlback type 1 changes (narrowing of joint space) in $3(13.6 \%)$, type II (obliteration of joint space) in $1(4.5 \%)$, and type III (bone attrition less than $5 \mathrm{~mm}$ ) in one (4.5\%). No type IV and $\mathrm{V}$ changes were found. Three patients had degenerative changes of the medial compartment, one of the patello-femoral joint, and one of both the medial and

Table 1. The assessment of symptom of the operated knees.

\begin{tabular}{|c|c|c|c|c|c|c|}
\hline \multirow[b]{2}{*}{$\begin{array}{l}\text { Instability } \\
\text { Pain }\end{array}$} & \multicolumn{2}{|c|}{ Pre-op } & \multicolumn{3}{|c|}{ Post-op } & \\
\hline & Yes & No & Yes & No & & \\
\hline Yes & $13(5$ & $.1 \%)$ & $4(18$ & & $1(4.5 \%)$ & $1(4.5 \%)$ \\
\hline No & $5(22$ & 7\%) & 0 & & $2(9.1 \%)$ & $18(81.8 \%)$ \\
\hline
\end{tabular}

Table 2. Correlation of symptom and Lysholm score with the duration from injury to surgery.

\begin{tabular}{lllll}
\hline Duration (months) & No. of case & positive & Symptom & Lysholm score \\
\hline$<3$ & 2 & 0 & $2(100 \%)$ & $92.3+/-3.9$ \\
$3-6$ & 14 & $2(14.2 \%)$ & $12(85.8 \%)$ & $86.1+/-9.4$ \\
$6-12$ & 4 & $1(25 \%)$ & $3(75 \%)$ & $68.4+/-8.6$ \\
$>12$ & 2 & $1(50 \%)$ & $1(50 \%)$ & $61.1+/-7.7$ \\
\hline
\end{tabular}

Table 3. The assessment of ligament laxity.

\begin{tabular}{|c|c|c|c|c|}
\hline & \multicolumn{4}{|c|}{ PCL laxity $(\mathrm{mm})$ by posterior drawer test } \\
\hline & 0 & $0-5$ & $5-10$ & $>10$ \\
\hline Pre-op & 0 & 0 & 0 & $22(100 \%)$ \\
\hline Post-op & $6(27.3 \%)$ & 12 (54.5\%) & $4(18.2 \%)$ & 0 \\
\hline
\end{tabular}


Acute augmentation for interstitial insufficiency of the posterior cruciate ligament. A two to five year clinical and radiographic study

patellofemoral compartments. In one case (4.5\%), preoperative degenerative changes had progressed at the latest follow-up.

Correlating the occurrence of degenerative changes with the interval time from injury to surgery (Tab. 4), we found a statistically significant correlation when patients had been operated on after more than 12 months from injury. The occurrence of degenerative changes was $13.6 \%(3 / 22)$ at 2-year follow-up and $22.7 \%(5 / 22)$ at 3 -year and longer follow-up $(\mathrm{P}<0.05)$, showing that the occurrence of degenerative changes is also positively correlated with duration of the follow up. When correlating degenerative changes with postoperative residual ligament laxity of the knee (Tab. 5), we found that the occurrence of osteoarthritic changes was $25 \%(3 / 12)$ for the knees presenting 0-5 mm laxity, 25\% (1/4) in those with $5-10 \mathrm{~mm}$ laxity, and $16.7 \%(1 / 6)$ in those knees with no residual PCL laxity. This difference was not statistically significant.

\section{Discussion}

The main finding of the present study is that arthroscopic augmentation of the PCL using transtibial single bundle procedure and autograft hamstring tendons provides satisfactory outcomes in $82 \%$ of patients at an average follow of 50 months after surgery. Outcomes are superior when performing surgery early. PCL reconstruction may be open or arthroscopic, using transtibial or tibial inlay procedures, one or double bundle ${ }^{12}$. This surgery may be compromised when ligament injuries are associated. The anatomy of the PCL is demanding to restore as the anatomical location of the femoral footprint may change, and it is unpredictably difficult to drill an anatomical femoral tunnel through the transtibial approach. In addition, when performing a transtbial technique, some tunnel enlargement, graft migration and elongation may occur over time ${ }^{13,14}$. On the other hand, the tibial inlay procedures has been conceived to prevent graft loosening, the 'killer turn' effect, and the graft friction, allowing to restore the native attachment of the PCL to the tibia ${ }^{15}$. However the results of transtibial procedures are comparable to those observed after tibial inlay surgery ${ }^{16,17}$. Up to date it is unknown to establish the best surgical procedure for PCL deficiency. We performed an arthroscopic augmentation using a single bundle transtibial procedure. Four-strand hamstring tendon autograft was used: it is easy to harvest, and provides suitable graft size and strong primary fixation strength ${ }^{18}$. In addition, we fixed the ligament on both the femoral and tibial sides to improve the stability of the construct ${ }^{19}$ and prevent progressive elongation of the graft $^{20}$. The sense to perform graft augmentation is to leave in situ the residual fibers of the PCL at both the attachment and insertion sites. We now point out that most PCL injuries occur with the knee flexed: in this position, the anterolateral bundle is stretched under tension, the postero-medial bundle is intact ${ }^{2,21-23}$. Such remnant fibers may heal with the graft and contribute to posterior knee stability ${ }^{15}$. The average Lysholm knee score was 86 points at final assessment compared to 49 points preoperatively. For what concerns the clinical symptoms evaluated with IKDC scores, Chen et al. found that $83 \%$ of their patients reported minimal pain or swelling during strenuous or moderate activities after reconstruction ${ }^{24}$. Considering the rate of normal (A)/nearly normal (B) IKDC outcomes as primary end point, a recent systematic review has reported an average success rate of $89.8 \%$ (SD 4.2 ) rate was after PCL augmentation, and $80.1 \%$ after reconstruction. Our IKDC outcomes are slightly lower to those reported by Del Buono et al. ${ }^{25}$, but the follow up of the present study is longer, and almost all patients are athletes. Specifically, 16 patients $(72.7 \%)$ returned to pre-injury sport activity level, and $3(13.6 \%)$ returned to a lower level. This procedure markedly reduces the ligament laxity, which was less than $5 \mathrm{~mm}$ in 18 of 22 $(81.8 \%)$ knees. PCL augmentation also provides lower complication rates compared to reconstruction procedures. Even though postoperative limitation in ROM may be a consequence after using hamstring tendon grafts ${ }^{12,25}$, only 1 patient reported postoperative limitation in range of motion. The completely recovered after manipulation under anesthesia.

Chronic PCL deficiency may induce the development of secondary degenerative changes ${ }^{1,5,6,9,26,27}$, mostly

Table 4: Correlation of osteoarthritis with duration of injury and follow-up time.

\begin{tabular}{lll}
\hline Duration of PCL injury (months) & $<2$ years follow-up & $>3$ years follow-up \\
\hline$<3(2)$ & $0 / 2(0 \%)$ & $0 / 2(0 \%)$ \\
$3-6(14)$ & $1 / 14(7.1 \%)$ & $3 / 14(21.4 \%)$ \\
$6-12(4)$ & $1 / 4(25 \%)$ & $1 / 4(25 \%)$ \\
$>12(2)$ & $1 / 2(50 \%)$ & $1 / 2(50 \%)$ \\
\hline
\end{tabular}

Table 5. The correlation of post-operative residual PCL laxity and secondary osteoarthritis.

\begin{tabular}{llll}
\hline PCL laxity $(\mathrm{mm})$ & 0 & $0-5$ & $5-10$ \\
OA change & $16.7 \%(1 / 6)$ & $25 \%(3 / 12)$ & $25 \%(1 / 4)$
\end{tabular}



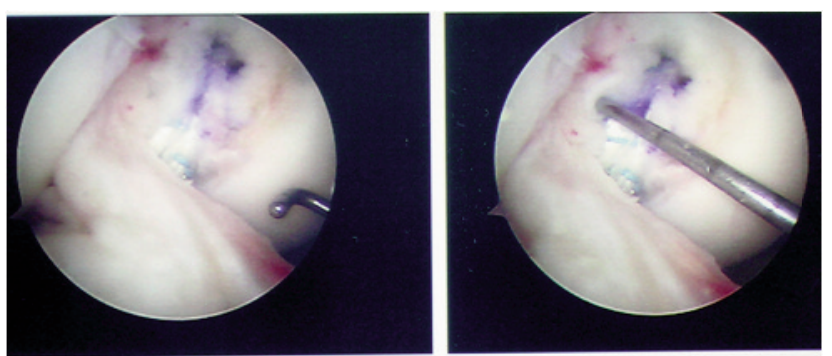

Figure 2. Preserved PCL substance with reconstructed graft.
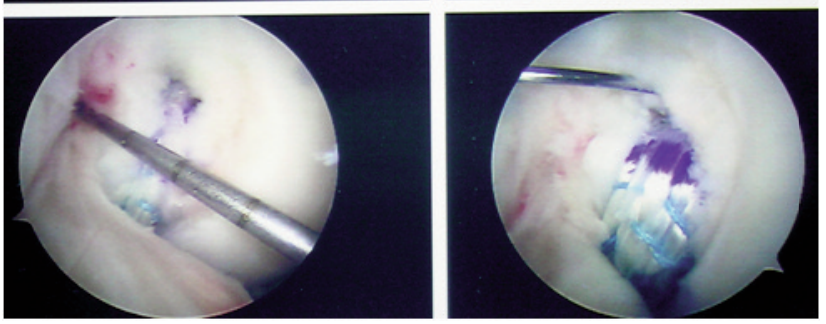

in the patellofemoral (20\%) and tibiofemoral compartments $(70 \%)$, accounting for an overall $46 \%$ occurrence of degenerative changes. We observed an overall incidence of osteoarthritic changes of $22.7 \%$. This relatively lower rate could be secondary to the preservation of $\mathrm{PCL}$ fibers ${ }^{15}$. Another reason to preserve $P C L$ fibers is to maintain the mechanoreceptors on the surface of the ligament's femoral and tibial attachment sites, which function as knee stabilizers, explaining why patients with posterior instability develop degenerative changes more slowly than those with anterior instability ${ }^{28}$. The occurrence of these changes correlated positively with the time from injury to surgery, and with the length of the follow-up, not with the post-operative PCL laxity.

The described PCL reconstruction technique has several merits. First, this procedure, differently from the inlay procedure, is safe for the posterior capsule, and, as suggested by Fanelli26, the posteromedial incision allows protection of the vascular structures. Hamstrings autografts are safe and, when whip stitched with strong non-absorbable material along the full length of the quadrupled graft, a strong bundle is produced. Second, by preserving the residual vascularized substance of the PCL, the augmented ligament is revascularised, with higher chances to heal (Fig. 2). At the tibial footprint, the residual substance of the PCL may protect the graft from the tibial 'killer turn' effect. Third, a double fixation, at both femoral and tibial side, is strong and secure, and allows aggressive rehabilitation and early recovery of motion.

As reported in previous studies, it is difficult to completely eliminate the posterior laxity of the knee $7,9,29,30$. We found up to $5 \mathrm{~mm}$ of laxity in $81 \%$ of patients, confirming that it is not necessary to completely restore the knee stability to achieve satisfactory results.

This study has several limitations: (1) no control group was included: a comparative study is needed to confirm the superiority of this augmentation tech- nique over reconstruction; (2) the follow-up period was probably sufficient to assess the presence of degenerative changes in the mid-term, but longer follow-ups are needed; (3) the presence of associated lesions may have biased results, but no statistically significant differences were found in Lysholm score, IKDC evaluation, and KT-1000 arthrometer results between patients with and without associated injuries. The strengths are a single-surgeon consecutive series with clear inclusion and exclusion criteria, a homogeneous standardized rehabilitation programme and standardized review programme with $100 \%$ follow up.

All the procedures described in the present study comply with the Muscles, Ligaments and Tendons Journal. Basic principles and recommendations in clinical and field science research ${ }^{31}$.

\section{Conclusions}

This procedure is effective in $82 \%$ after an average of 4 years from the index surgery. Early surgery improves outcome and also reduces the occurrence of degenerative changes.

Authors' contribution: TC and CK conceived the study. TC and CK advised on the practicalities of the surgery. TC and CK performed all surgical procedures, were involved in the clinical assessment, performed the review of the literature and wrote the initial draft. NM coordinated the work implant in all its phases. ADB and NM wrote the definitive version of the manuscript, and corrected the English form. All Authors read and approved the final manuscript.

\section{Conflict of interests}

The Authors declare that they have no conflict of interests regarding the publication of this paper. 
Acute augmentation for interstitial insufficiency of the posterior cruciate ligament. A two to five year clinical and radiographic study

\section{References}

1. Dandy DJ, Pusey RJ. The long-term results of unrepaired tears of the posterior cruciate ligament. J Bone Joint Surg Br. 1982;64:92-94.

2. Fowler PJ, Messieh SS. Isolated posterior cruciate ligament injuries in athletes. Am J Sports Med. 1987;15:553-557.

3. Shino K, Horibe S, Nakata K, Maeda A, Hamada M, Nakamura N. Conservative treatment of isolated injuries to the posterior cruciate ligament in athletes. J Bone Joint Surg Br. 1995; 77:895-900.

4. Osti L, Papalia R, Del Buono A, Leonardi F, Denaro V, Maffulli N. Surgery for ACL deficiency in patients over 50. Knee Surg Sports Traumatol Arthrosc. 2011;19:412-417.

5. Clancy WG, Shelbourne KD, Zoellner GB, Keene JS, Reider B, Rosenberg TD. Treatment of knee joint instability secondary to rupture of the posterior cruciate ligament. Report of a new procedure. J Bone Joint Surg Am. 1983;65:310-322.

6. Cross MJ, Powell JF. Long-term followup of posterior cruciate ligament rupture: a study of 116 cases. Am J Sports Med. 1984;12:292-297.

7. Lipscomb AB, Jr., Anderson AF, Norwig ED, Hovis WD, Brown $\mathrm{DL}$. Isolated posterior cruciate ligament reconstruction. Longterm results. Am J Sports Med. 1993;21:490-496.

8. Osti L, Papalia R, Del Buono A, Merlo F, Denaro V, Maffulli N. Simultaneous surgical management of chronic grade-2 valgus instability of the knee and anterior cruciate ligament deficiency in athletes. Knee Surg Sports Traumatol Arthrosc. 2010;18: 312-316.

9. Richter M, Kiefer H, Hehl G, Kinzl L. Primary repair for posterior cruciate ligament injuries. An eight-year follow-up of fiftythree patients. Am J Sports Med. 1996;24:298-305.

10. Malek MM, Fanelli GC. Technique of arthroscopically assisted PCL reconstruction. Orthopedics. 1993;16:961-966.

11. Ahlback S. Osteoarthrosis of the knee. A radiographic investigation. Acta Radiol. 1968;Suppl 277:277-272.

12. Papalia R, Osti L, Del Buono A, Denaro V, Maffulli N. Tibial inlay for posterior cruciate ligament reconstruction: a systematic review. Knee. 2010;17:264-269.

13. Cooper DE, Stewart D. Posterior cruciate ligament reconstruction using single-bundle patella tendon graft with tibial inlay fixation: 2- to 10-year follow-up. Am J Sports Med. 2004;32:346360.

14. Loos WC, Fox JM, Blazina ME, Del Pizzo W, Friedman MJ. Acute posterior cruciate ligament injuries. Am J Sports Med. 1981;9:86-92.

15. Ahn JH, Yang HS, Jeong WK, Koh KH. Arthroscopic transtibial posterior cruciate ligament reconstruction with preservation of posterior cruciate ligament fibers: clinical results of minimum 2-year follow-up. Am J Sports Med. 2006;34:194-204.

16. Deehan DJ, Salmon LJ, Russell VJ, Pinczewski LA. Endoscopic single-bundle posterior cruciate ligament reconstruc- tion: results at minimum 2-year follow-up. Arthroscopy. 2003; 19:955-962.

17. Wang CJ, Chen HS, Huang TW. Outcome of arthroscopic single bundle reconstruction for complete posterior cruciate ligament tear. Injury. 2003;34:747-751.

18. Toritsuka Y, Horibe S, Mitsuoka T, Nakamura N, Hamada M, Shino K. Comparison between the cross-sectional area of bone-patellar tendon-bone grafts and multistranded hamstring tendon grafts obtained from the same patients. Knee Surg Sports Traumatol Arthrosc. 2003;11:81-84.

19. Chen $\mathrm{CH}$, Chou SW, Chen WJ, Shih $\mathrm{CH}$. Fixation strength of three different graft types used in posterior cruciate ligament reconstruction. Knee Surg Sports Traumatol Arthrosc. 2004; 12:371-375.

20. Chen $\mathrm{CH}$, Chen WJ, Shih $\mathrm{CH}$. Arthroscopic reconstruction of the posterior cruciate ligament with quadruple hamstring tendon graft: a double fixation method. J Trauma. 2002;52:938945.

21. Harner CD, Hoher J. Evaluation and treatment of posterior cruciate ligament injuries. Am J Sports Med. 1998;26:471-482.

22. Peterson CA, Warren RF. Management of acute and chronic posterior cruciate ligament injuries. New approaches. Am J Knee Surg. 1996;9:172-184.

23. Race A, Amis AA. The mechanical properties of the two bundles of the human posterior cruciate ligament. J Biomech. 1994:27:13-24.

24. Chen $\mathrm{CH}$, Chuang TY, Wang KC, Chen WJ, Shih CH. Arthroscopic posterior cruciate ligament reconstruction with hamstring tendon autograft: results with a minimum 4-year followup. Knee Surg Sports Traumatol Arthrosc. 2006;14:10451054.

25. Del Buono A, Radmilovic J, Gargano G, Gatto S, Maffulli N. Augmentation or reconstruction of $\mathrm{PCL}$ ? A quantitative review. Knee Surg Sports Traumatol Arthrosc. 2013;21:1050-1063.

26. Fanelli GC, Edson CJ. Posterior cruciate ligament injuries in trauma patients: Part II. Arthroscopy. 1995;11:526-529.

27. Keller PM, Shelbourne KD, McCarroll JR, Rettig AC. Nonoperatively treated isolated posterior cruciate ligament injuries. Am J Sports Med. 1993;21:132-136.

28. Safran MR, Allen AA, Lephart SM, Borsa PA, Fu FH, Harner $\mathrm{CD}$. Proprioception in the posterior cruciate ligament deficient knee. Knee Surg Sports Traumatol Arthrosc. 1999;7:310-317.

29. Fleming RE, Blatz DJ, McCarroll JR. Posterior problems in the knee. Posterior cruciate insufficiency and posterolateral rotatory insufficiency. Am J Sports Med. 1981;9:107-113.

30. Noyes FR, Barber-Westin SD. Surgical restoration to treat chronic deficiency of the posterolateral complex and cruciate ligaments of the knee joint. Am J Sports Med. 1996;24:415426.

31. Padulo J, Oliva F, Frizziero A, Maffulli N. Muscles, Ligaments and Tendons Journal. Basic principles and recommendations in clinical and field science research. MLTJ. 2013;3(4):250-252. 